\title{
ARTIGO
}

\section{FERRAMENTA COMPUTACIONAL DIDÁTICA PARA SIMULAÇÃO DE MÁQUINAS ELÉTRICAS DE CORRENTE CONTÍNUA EM CONEXÃO COMPOSTA COM DERIVAÇÃO LONGA ${ }^{1}$}

\author{
Ana Carolina Silva Castro ${ }^{2}$ \\ Mariana Guimarães dos Santos \\ Patrick Santos de Oliveira \\ Ana Paula Lima dos Santos \\ Gustavo Lobato Campos
}

\section{RESUMO}

As máquinas elétricas estão presentes em quase todas as atividades que envolvem a manipulação de cargas em ambientes industriais, comerciais e residenciais. Assim, o estudo do funcionamento destes dispositivos é fundamental para diversos cursos de graduação, como o curso de engenharia elétrica e o de controle e automação, por exemplo. Neste contexto, a associação entre os conhecimentos teóricos e práticos auxilia no estudo dos alunos e na forma de lecionar dos professores. No entanto, muitas vezes não é possível realizar todas as situações teóricas nos laboratórios. Nestes casos, a utilização da simulação computacional surge como uma ferramenta eficiente, capaz de apresentar as curvas fundamentais para o estudo das máquinas de forma didática e para todas as condições de carga existentes. Desta forma, esse artigo apresenta resultados de simulação computacional de máquinas elétricas de corrente contínua (motores e geradores), realizada na ferramenta $M A T L A B \circledR$, na configuração composta em derivação longa. O modelo desenvolvido é baseado nas equações de regime permanente de tais equipamentos e o objetivo é obter curvas práticas, facilmente encontradas nas principais literaturas do ramo. A ferramenta computacional desenvolvida é validada por meio de comparação com curvas da literatura, apresentando concordância satisfatória.

Palavras-chave: Ferramenta didática. Máquinas elétricas de corrente contínua. MATLAB®.

\section{INTRODUÇÃO}

As máquinas elétricas são dispositivos conversores de energia que estão presentes em quase todas as aplicações industriais e atividades que envolvem manipulação de cargas. As máquinas podem ser classificadas, conforme o seu tipo de alimentação, em máquinas de

\footnotetext{
${ }^{1}$ Como inserir este artigo: CASTRO, Ana Carolina Silva et al. Ferramenta computacional didática para simulação de máquinas elétricas de corrente contínua em conexão composta com derivação longa. ForScience: revista científica do IFMG, Formiga, v. 7, n. 1, e00584, jan./jun. 2019. DOI: 10.29069/forscience.2019v7n1.e584.
}

${ }^{2}$ Autor para correspondência: Ana Carolina Silva Castro. E-mail: anacarolinasilvacastro@yahoo.com.br. 
CASTRO, A. C. S. et al. Ferramenta computacional didática para simulação de máquinas elétricas de corrente contínua de conexão composta com derivação longa

corrente contínua ou máquinas de corrente alternada. Ambas encontram aplicações em diferentes atividades. No que diz respeito às máquinas elétricas, existe ainda uma classificação conforme o fluxo de energia da mesma, em motores (conversão de energia elétrica em mecânica) ou geradores (conversão da energia mecânica em elétrica). Além disso, tanto os motores quanto os geradores de corrente contínua podem ser classificados conforme a alimentação do seu enrolamento de campo em: excitação independente, série, shunt e composto cumulativo ou diferencial (KOSOW, 2000; FITZGERALD; KINGSLEY; UMANS, 2014).

Devido a sua versatilidade, as máquinas de corrente contínua encontram grande aplicação, principalmente como motores, em atividades como laminação de metal, indústria têxtil, prensas, veículos de tração, guinchos e guindastes, dentre outros (DEL TORO, 1999).

O estudo das máquinas de corrente contínua engloba conhecimentos a respeito do seu princípio de funcionamento, tipos de acionamentos e avaliação de suas características para diferentes condições de carga. Muitas vezes, o ensino de conhecimentos desta natureza é de difícil compreensão por parte dos alunos, principalmente no que diz respeito à associação entre conhecimentos teórico e prático (FARIAS, 2017).

O modelo de ensino tradicional adotado na educação em engenharia e encontrado na maioria das Universidades é fortemente dependente do professor, das técnicas de ensino e dos recursos institucionais. Na busca por técnicas de ensino e de aprendizagem mais eficientes, os professores buscam ensinar os conceitos teóricos associados a habilidades práticas e, neste contexto, o uso de simulação computacional como ferramenta pedagógica está cada vez mais presente nas práticas docentes, possibilitando uma dinâmica de ensino que promove um aprendizado mais significativo e motivador (LODER; BENDER, 2007; KHALIL, 2012).

$\mathrm{Na}$ engenharia elétrica, as ferramentas computacionais são muito utilizadas para simulação de problemas e na modelagem de equipamentos e sistemas. No entanto, muitas vezes, essas ferramentas não são exploradas como método de aprendizagem nas universidades, sendo aplicadas em poucas disciplinas do curso. Uma das razões que limita a utilização de tais ferramentas é que o desenvolvimento da modelagem dos equipamentos e sistemas requer um tempo de estudo que normalmente é incompatível com a carga horária e ementa das disciplinas. Assim, o desenvolvimento prévio de ferramentas computacionais capazes de simular o comportamento de sistemas e equipamentos para diferentes situações pode tornar a utilização da simulação computacional uma ferramenta de aprendizagem viável para algumas disciplinas (MARCHETTO FILHO; PINHEIRO; CARATI, 2016). Neste caso, 
CASTRO, A. C. S. et al. Ferramenta computacional didática para simulação de máquinas elétricas de corrente contínua de conexão composta com derivação longa

a modelagem de tais dispositivos permitiria ao aluno uma visualização do comportamento das referidas máquinas em situações que não são possíveis em laboratório. Além disso, é possível confrontar os conhecimentos teóricos, com as medições práticas de bancada e com os resultados obtidos pela simulação computacional. Esse tipo de abordagem, além de desenvolver habilidades computacionais, estimula e motiva a aprendizagem dos alunos (BELHOT; FIGUEIREDO; MALAVÉ, 2001; PEREIRA, 2016).

Diante do exposto, este artigo apresenta uma modelagem e os resultados de simulações computacionais do funcionamento de geradores e motores de corrente contínua na configuração composta em derivação longa. Vale ressaltar, que a intenção do modelo está na operação de tais dispositivos em regime permanente e na extração de curvas características práticas encontradas nas bibliografias utilizadas para o ensino destas máquinas. A maioria dos artigos encontrados na literatura utiliza o modelo da máquina de corrente contínua no domínio do tempo e apresenta resultados de curvas dinâmicas, formas de onda de tensões, torques e correntes (MELO, 2006; CRESPO; GUINGLA, 2010; ALVARADO, 2012; YILDIZ, 2012). Tais resultados são relevantes para o estudo das máquinas elétricas de corrente contínua, no entanto, para o ambiente de sala de aula na graduação, as curvas obtidas neste artigo mostramse mais adequadas.

\section{SIMULAÇÃO DE MÁQUINAS ELÉTRICAS DE CORRENTE CONTÍNUA}

\subsection{Desenvolvimento da Modelagem Matemática}

Para o desenvolvimento da modelagem matemática da máquina de Corrente Contínua (CC) shunt e excitação independente, operando como motor e como gerador, considera-se a indutância no circuito equivalente desprezível, uma vez que, em regime permanente e excitação com corrente contínua, a indutância pode ser modelada como um curto-circuito (HAMBLEY, 2014).

A Equação (1) apresenta a relação entre as tensões induzidas na armadura e suas correspondentes velocidades de operação, para distintas condições. Esta equação explicita a proporcionalidade entre as duas grandezas, tornando-se essencial para a modelagem de todas configurações de máquinas CC consideradas.

$$
\frac{E_{A 1}}{E_{A 2}}=\frac{n_{1}}{n_{2}}
$$


CASTRO, A. C. S. et al. Ferramenta computacional didática para simulação de máquinas elétricas de corrente contínua de conexão composta com derivação longa

$\mathrm{Na}$ Equação (1): $E_{A 1}$ representa a tensão de magnetização na velocidade $n_{1}$ e $E_{A 2}$ representa a tensão de magnetização na velocidade $n_{2}$ (HAMBLEY, 2014).

Adicionalmente, as Equações (2) e (3) são utilizadas como base para o desenvolvimento da modelagem de geradores e motores CC. A Equação (2) relaciona a tensão induzida na armadura com a velocidade operação da máquina, enquanto a Equação (3) relaciona a corrente de armadura com o torque desenvolvido.

$$
\begin{aligned}
& E_{A}=K \emptyset w_{m} \\
& T_{D e s}=K \emptyset I_{A}
\end{aligned}
$$

Nas Equações (2) e (3): $K$ é uma constante que depende dos parâmetros de projeto da máquina, $\varnothing$ é o fluxo magnético produzido por cada polo do estator, $w_{m}$ é a velocidade angular do rotor, $T_{D e s}$ é o torque desenvolvido e $I_{A}$ é a corrente de armadura (HAMBLEY, 2014).

Em específico, para o gerador composto, a modelagem envolve, além da comunicação entre equações, uma análise gráfica para definir os valores terminais. Isto ocorre porque, nestas máquinas, a tensão de saída depende diretamente da entrada de corrente de campo.

O restante das equações utilizadas no modelo é definido de acordo com o tipo de máquina em questão e apresentado a seguir.

\subsubsection{Modelagem dos Motores Compostos Cumulativo e Diferencial}

A Figura 1 apresenta o circuito equivalente do motor de corrente contínua composto com derivação longa, por meio do qual foi extraída a Equação (7).

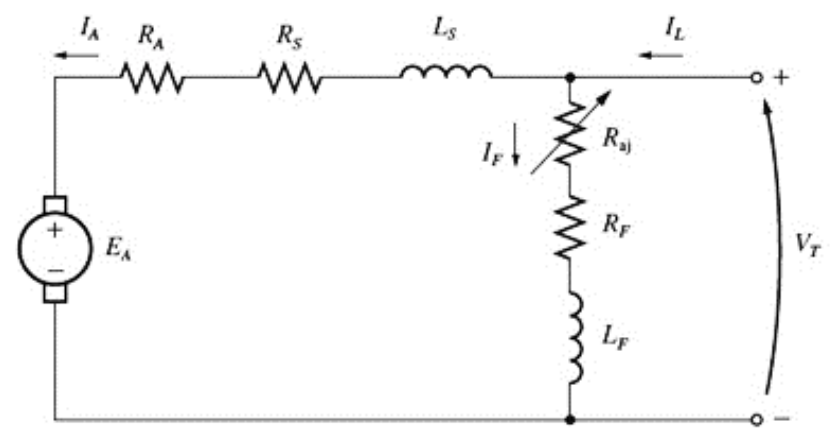

Figura 1 - Circuito equivalente do motor composto com derivação longa Fonte: Chapman (2013). 
CASTRO, A. C. S. et al. Ferramenta computacional didática para simulação de máquinas elétricas de corrente contínua de conexão composta com derivação longa

$$
E_{A}=V_{T}-I_{A}\left(R_{A}+R_{S}\right)
$$

A Equação (8) calcula a corrente de campo pela relação entre a tensão terminal e a resistência de campo shunt. Já a Equação (9), utiliza $I_{F}$ e o efeito da relação entre os campos série e derivação para a obtenção da corrente de campo efetiva do motor composto.

$$
\begin{gathered}
I_{F}=\frac{V_{T}}{R_{F}} \\
I_{e q}=\frac{N_{S E}}{N_{F}} I_{A} \\
I_{F}^{*}=I_{F} \pm I_{e q}
\end{gathered}
$$

Nas Equações (9) e (10): $I_{e q}$ é a corrente equivalente do campo em derivação, $I_{F}^{*}$ é a corrente efetiva total do campo em derivação e $N_{F}$ é o número de espiras por polo no enrolamento shunt (CHAPMAN, 2013).

Para o motor composto cumulativo, a corrente de campo efetiva é calculada considerando-se o sinal positivo da Equação (10), enquanto para o motor diferencial, considera-se o sinal negativo.

As Equações (7) e (10) representam a chave para a modelagem dos motores de corrente contínua compostos cumulativo e diferencial. Quando associadas à Equação (1), proporcionam os parâmetros necessários para substituição na Equação (6) e fornecimento dos valores de saída da máquina.

\subsubsection{Modelagem dos Geradores Compostos Cumulativo e Diferencial}

A tensão terminal do gerador de corrente contínua composto com derivação longa pode ser obtida conforme mostra a Equação (12), extraída da Figura 2, que apresenta o circuito equivalente da máquina.

$$
V_{T}=E_{A}-I_{A}\left(R_{A}+R_{S}\right)
$$


CASTRO, A. C. S. et al. Ferramenta computacional didática para simulação de máquinas elétricas de corrente contínua de conexão composta com derivação longa

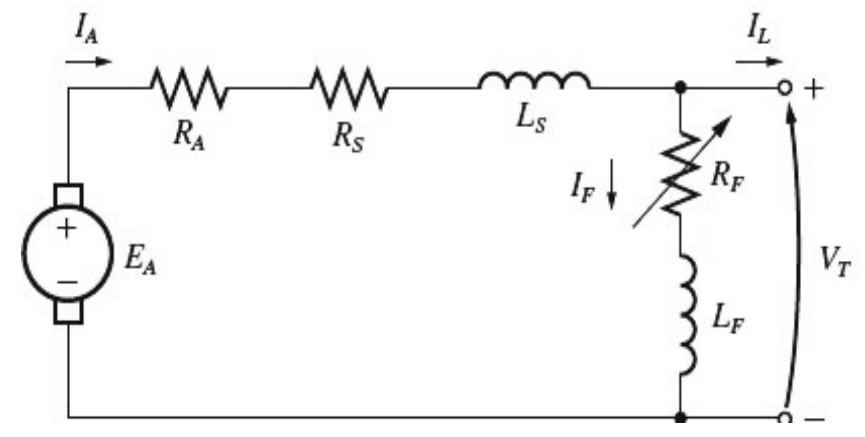

Figura 2 - Circuito equivalente do gerador composto com derivação longa Fonte: Chapman (2013).

A análise do gerador composto para obtenção de sua característica de saída não é realizada apenas por meio da comunicação entre as equações base de sua modelagem, é necessária também uma análise gráfica, devido ao fato de que os parâmetros de entrada do gerador composto dependem diretamente da saída (a corrente de campo shunt depende da tensão terminal).

No gerador composto, a queda de tensão total nas resistências série e de armadura depende da corrente de armadura, assim como a corrente equivalente $I_{e q}$, definida na Equação (9). Ambas formam os dois lados de um triângulo no gráfico que relaciona a curva de magnetização, na velocidade de acionamento considerada, e a curva linear de $R_{F}$, como apresentado na Figura 3. O ponto onde este triângulo se encaixa perfeitamente entre a reta da resistência shunt e a curva de magnetização determina a tensão terminal do gerador.

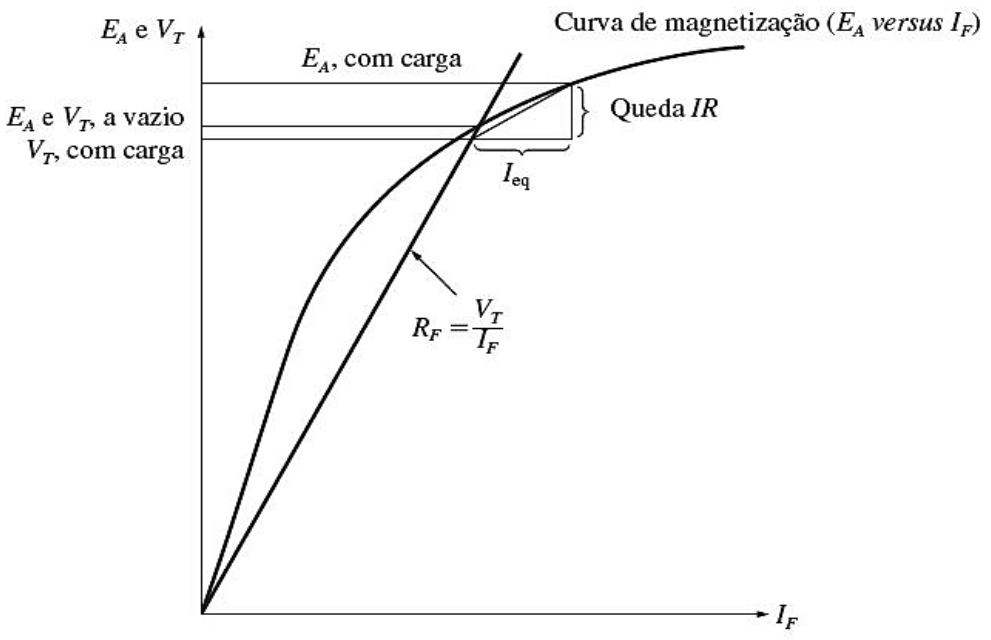

Figura 3 - Análise gráfica do gerador composto cumulativo Fonte: Chapman (2013).

A única diferença matemática na análise do gerador composto cumulativo e do gerador composto diferencial consta no sinal considerado para a corrente $I_{e q}$. No composto 
CASTRO, A. C. S. et al. Ferramenta computacional didática para simulação de máquinas elétricas de corrente contínua de conexão composta com derivação longa

cumulativo, esta corrente é positiva, somada ao valor de $I_{F}$ para a obtenção da corrente efetiva total e, consequentemente, a base do triângulo se direciona para a direita, como ilustra a Figura 3. Já no gerador diferencial, a corrente $I_{e q}$ é negativa e, quando somada ao valor de $I_{F}$ para a obtenção da corrente efetiva total, a base triângulo se direciona para a esquerda, como apresenta-se na Figura 4.

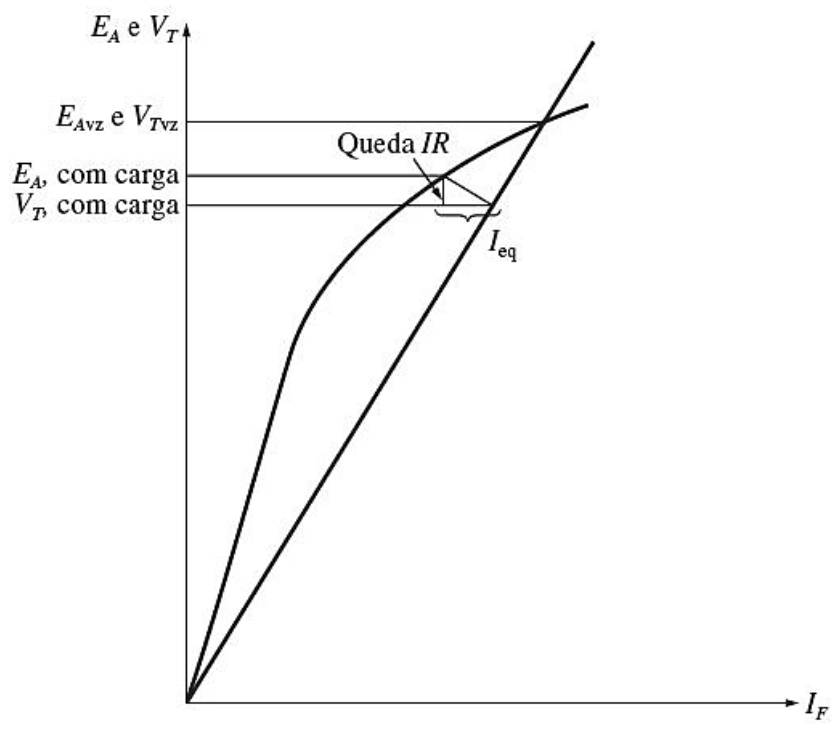

Figura 4 - Análise gráfica do gerador composto diferencial Fonte: Chapman (2013).

As Figuras 5 e 6 ilustram a forma como é realizada a construção gráfica da curva característica de saída do gerador composto e do gerador diferencial, respectivamente. Em ambas as figuras são possíveis observar a cada ponto no qual o triângulo de teste se encaixa, a tensão $V_{T}$ é coletada e definida como a tensão terminal naquele ponto. Na Figura 6 ainda é evidenciada a diferença de decaimento entre a curva do gerador em derivação e do composto diferencial.

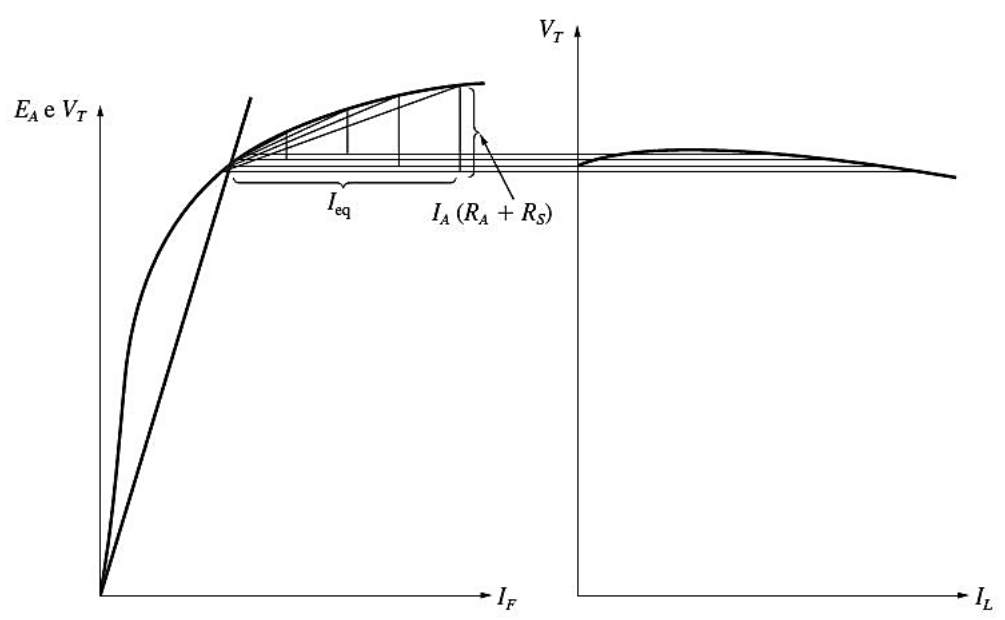

Figura 5 - Obtenção gráfica da tensão de saída do gerador composto cumulativo Fonte: Chapman (2013). 
CASTRO, A. C. S. et al. Ferramenta computacional didática para simulação de máquinas elétricas de corrente contínua de conexão composta com derivação longa

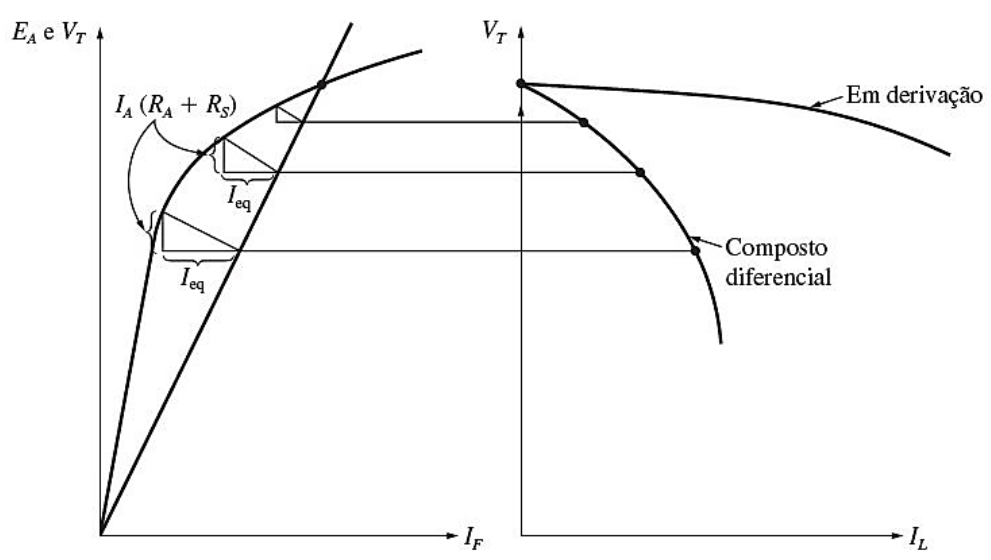

Figura 6 - Obtenção gráfica da tensão de saída do gerador composto diferencial Fonte: Chapman (2013).

Toda a modelagem matemática utilizada é baseada em equações e observações apresentadas em (CHAPMAN, 2013; HAMBLEY, 2014), ambas referências modernas e conceituadas na área.

\subsection{Implementação Computacional}

No código desenvolvido, primeiramente é necessário se carregar um arquivo em Excel com os dados da curva de magnetização da máquina (incluindo correntes de campo, tensões de magnetização e a velocidade na qual a curva foi obtida e a inserção dos dados de entrada específicos para cada configuração de máquina). Em seguida, o algoritmo faz a conexão entre as equações citadas na modelagem de cada máquina a fim de imprimir a curva característica de saída. No caso do gerador composto, além da comunicação entre as equações de modelagem, faz-se necessária a realização de uma análise gráfica pelo código, tornando a programação mais complexa.

A seguir, será detalhado o procedimento para a implementação computacional de cada máquina.

\subsubsection{Algoritmo dos Motores Compostos Cumulativo e Diferencial}

Os dados de entrada consistem na curva de magnetização, tensão de entrada, resistência de armadura, resistência de campo série, resistência de campo shunt, número de espiras por polo no enrolamento de campo série, número de espiras por polo no enrolamento 
CASTRO, A. C. S. et al. Ferramenta computacional didática para simulação de máquinas elétricas de corrente contínua de conexão composta com derivação longa

de campo shunt, assim como um vetor de valores de corrente de armadura representando a variação de carga.

Primeiramente, a corrente do campo em derivação é encontrada por meio da equação (8). Em seguida, o valor obtido é somado, no caso do motor composto, ou subtraído, no caso do motor composto diferencial, às correntes equivalentes, definidas na Equação (9), correspondentes a cada valor de corrente de armadura, para se obter as correntes efetivas de campo. Estas correntes são interpoladas na curva de magnetização, para que sejam encontradas as tensões induzidas de armadura correspondentes $\left(E_{A 0}\right)$.

Com a Equação (7), encontram-se os valores de tensão de magnetização $\left(E_{A}\right)$ para cada valor de corrente de armadura. Estes dados são inseridos em $E_{A 1}$ na Equação (1) para que sejam encontradas as velocidades de operação correspondentes a cada valor de tensão, sendo que em $\mathrm{n}_{2}$ é inserida a velocidade na qual foi obtida a curva de magnetização e em $E_{A 2} \mathrm{o}$ valor de $E_{A 0}$

Por fim, os dados de entrada pertinentes, assim como cada velocidade encontrada, são inseridos na Equação (6) e o torque desenvolvido correspondente a cada velocidade é determinado.

A Figura 7 apresenta um fluxograma que resume o passo a passo da implementação computacional descrita anteriormente.

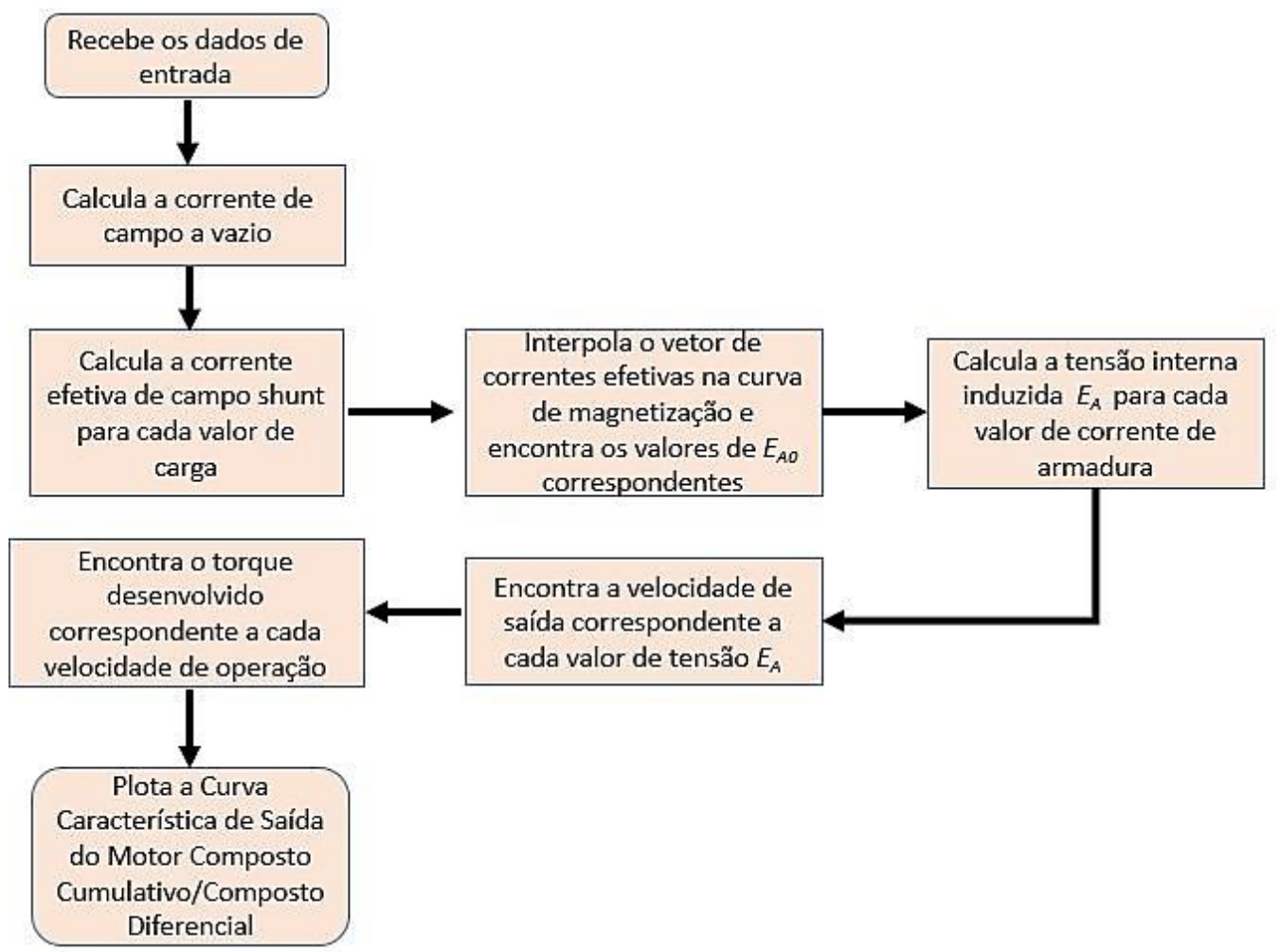

Figura 7 - Fluxograma do algoritmo dos motores compostos cumulativo e diferencial desenvolvido

Fonte: Autores (2018). 
CASTRO, A. C. S. et al. Ferramenta computacional didática para simulação de máquinas elétricas de corrente contínua de conexão composta com derivação longa

\subsubsection{Algoritmo dos Geradores Compostos Cumulativo e Diferencial}

Os dados de entrada para os geradores composto cumulativo e composto diferencial mostram-se os mesmos considerados anteriormente para os motores CC compostos, exceto no parâmetro tensão de entrada, que é substituído pela velocidade de acionamento.

Primeiramente, com a Equação (9), são calculados os valores de corrente equivalente para cada valor de carga, ou seja, para cada valor de corrente de armadura presente no vetor de entrada. Em seguida, todos os valores de tensão induzida da curva de magnetização carregada são adaptados na velocidade de acionamento do gerador.

Em um primeiro laço de iteração, calcula-se um vetor que contém as tensões terminais hipotéticas que resultam do produto entre a resistência de campo e as correntes de campo da curva de magnetização (curva linear da Figura 3). Em seguida, outro vetor recebe a diferença entre as tensões de magnetização e as tensões terminais hipotéticas, $E_{A^{-}} V_{T}$, calculadas anteriormente.

Na sequência, calcula-se um vetor com produto entre a soma das resistências de armadura e de campo série e todas as correntes de armadura consideradas, representando a queda $\left(R_{A}+R_{S}\right) I_{A}$.

No loop principal do algoritmo computacional, é realizada uma varredura para o teste de cada valor de corrente armadura inserido no vetor de entrada. Interno a este laço, há um outro responsável por fazer a varredura para o teste de cada valor de corrente de campo. Por fim, dentro do loop anterior, há diversas condicionais que resultam no valor de tensão terminal do gerador.

A primeira condicional analisa a diferença entre as curvas de forma a detectar a tensão a vazio da máquina. Teoricamente, esta tensão encontra-se no ponto de intersecção entre as duas curvas. No algoritmo, assume-se que o primeiro ponto onde a queda na resistência de armadura mostra-se menor que a diferença entre as curvas, é o ponto de tensão a vazio.

Nas próximas condicionais, são avaliados em quais pontos do gráfico, somando-se (composto cumulativo) ou subtraindo-se (composto diferencial) a corrente equivalente da corrente de campo $I_{F}$, o valor da queda $\left(R_{A}+R_{S}\right) I_{A}$ encontra-se entre os valores da diferença entre as curvas. Quando estes pontos são detectados, faz-se uma interpolação e o valor da tensão terminal é definido. A diferença primordial na implementação dos dois tipos de motores encontra-se no sinal considerado para a soma da corrente equivalente, sendo que no composto cumulativo o sinal é positivo e no composto diferencial é o sinal negativo. 
CASTRO, A. C. S. et al. Ferramenta computacional didática para simulação de máquinas elétricas de corrente contínua de conexão composta com derivação longa

A Figura 8 mostra um fluxograma que resume a sequência de ações do código desenvolvido para a obtenção da curva característica de saída dos geradores compostos cumulativo e diferencial.

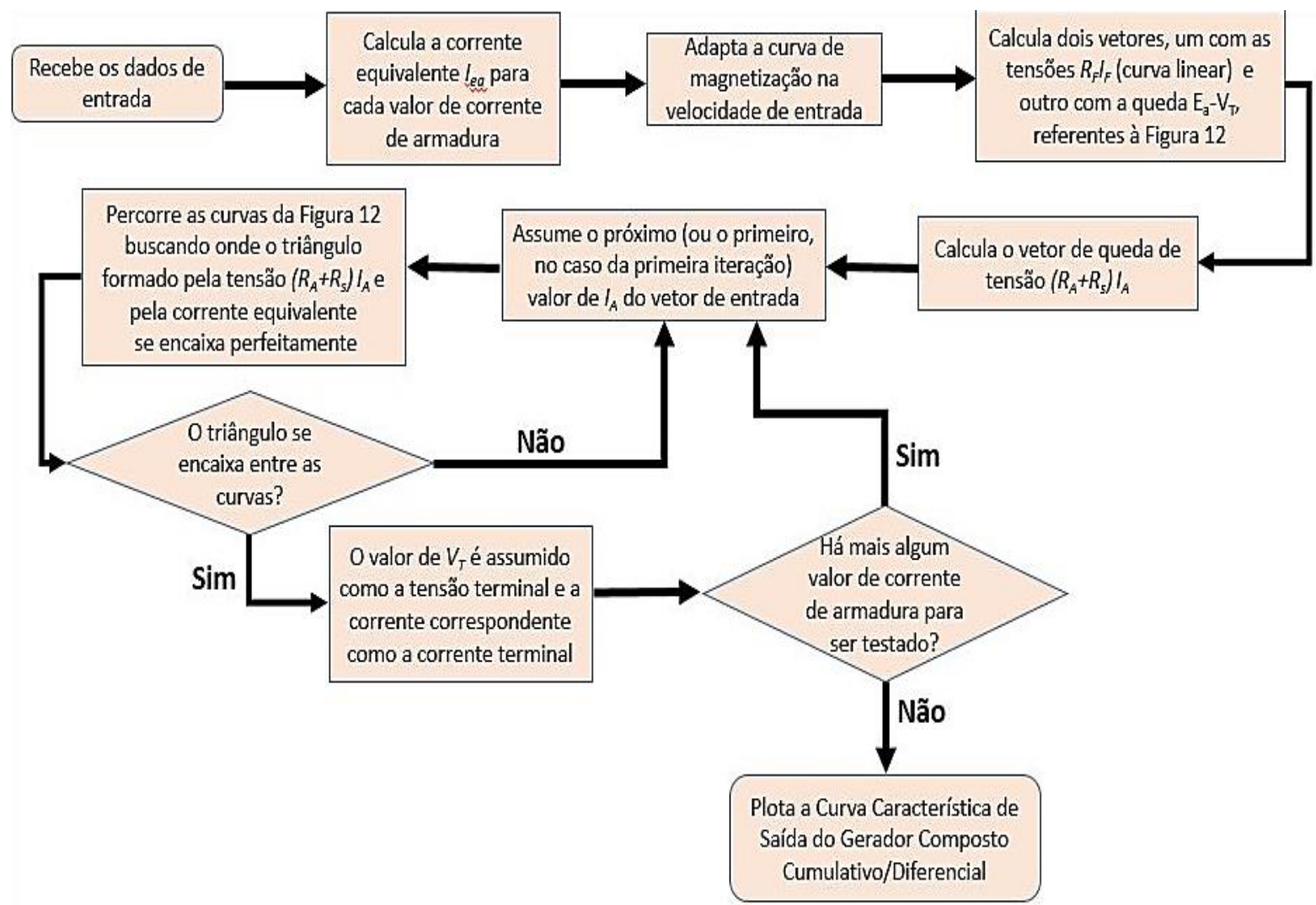

Figura 8 - Fluxograma do algoritmo dos geradores compostos cumulativo e diferencial desenvolvido Fonte: Autores (2018).

\subsection{Validação dos Resultados}

A validação dos resultados é realizada por meio da comparação com resultados encontrados nos livros texto referência deste assunto e com resultados de medições realizadas no laboratório de máquinas elétricas do Instituto Federal de Educação, Ciência e Tecnologia de Minas Gerais, Campus Formiga.

A resistência de armadura da máquina utilizada para teste e validação dos resultados é de $11,3 \Omega$ (com interpolo) e a resistência de campo é de $733,33 \Omega$. A corrente nominal da máquina, operando como gerador, é de 1,68 A, e a tensão nominal é de $220 \mathrm{~V}$. Quando configurada no modo gerador, a máquina foi acionada à 1800rpm e, no caso de excitação independente, submetida à $220 \mathrm{~V}$ no enrolamento de campo.

A seguir, são explicitadas as validações para cada uma das máquinas. 
CASTRO, A. C. S. et al. Ferramenta computacional didática para simulação de máquinas elétricas de corrente contínua de conexão composta com derivação longa

\subsubsection{Validação do Motor Composto Cumulativo}

O comportamento da curva de saída do motor composto cumulativo está presente na Figura 9. Na mesma figura, também está ilustrado o comportamento terminal do gerador shunt. Ambas foram ilustradas no mesmo gráfico a fim de tornar possível a melhor visualização do comportamento do motor composto cumulativo. Na condição sem carga (a vazio), as duas máquinas possuem a mesma velocidade. Com cargas pequenas, os comportamentos de ambas são semelhantes, pois a baixa drenagem da corrente de carga torna o efeito do enrolamento série pequeno. À medida que a carga aumenta, o efeito do campo série torna-se cada vez mais determinante, fazendo com que o restante da curva se assemelhe ao comportamento do motor série. Pode-se perceber que o motor composto cumulativo não dispara sua velocidade quando está sem carga (1800 rpm, a vazio) e possui grande desempenho de torque na partida (curva tendendo a valores muito mais altos que o motor shunt para velocidade nula).

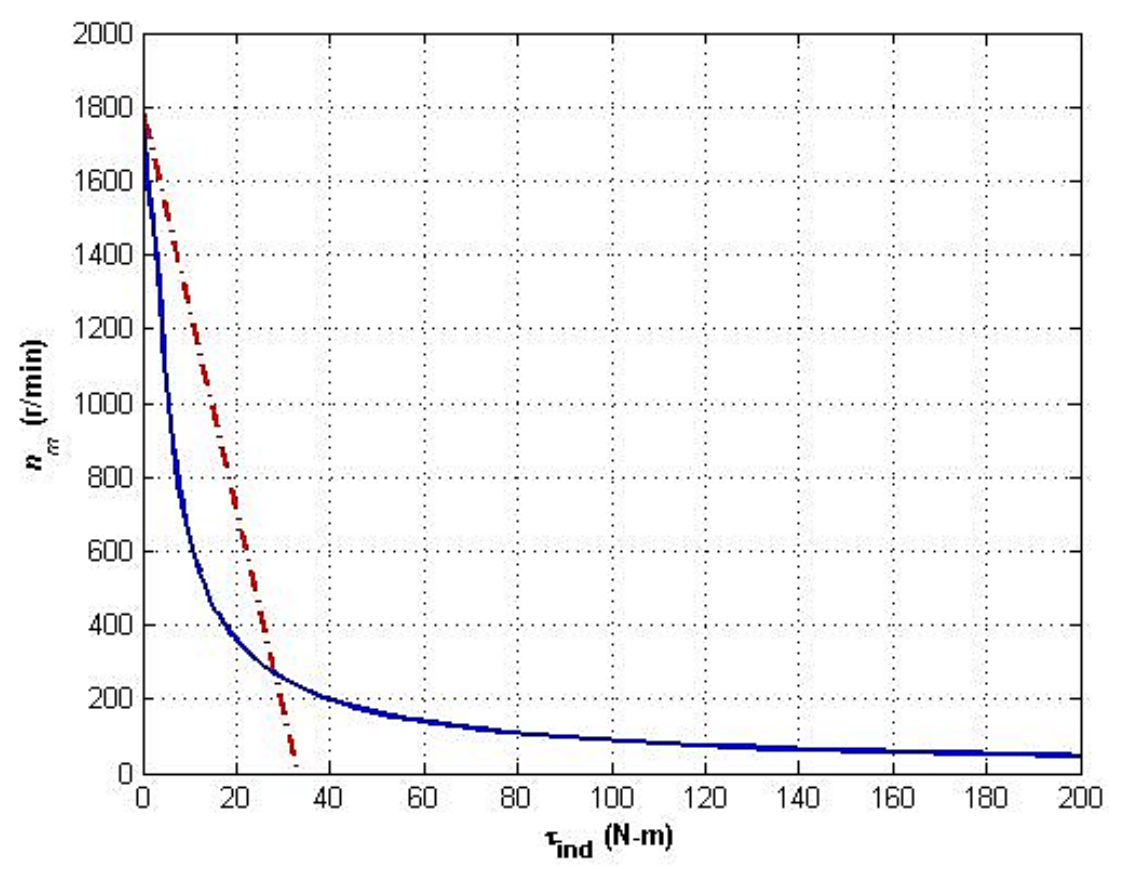

Figura 9 - Simulação das curvas de saída dos motores shunt (vermelha pontilhada) e composto cumulativo (azul contínua)

Fonte: Autores (2018).

A Figura 10 apresenta curvas genéricas de saída do motor derivação e do motor composto cumulativo, com a mesma velocidade a vazio. Comparando-se esta figura à Figura 
CASTRO, A. C. S. et al. Ferramenta computacional didática para simulação de máquinas elétricas de corrente contínua de conexão composta com derivação longa

9, constata-se a mesma característica comportamental. Assim, todas as constatações realizadas na simulação condizem com as previsões teóricas.

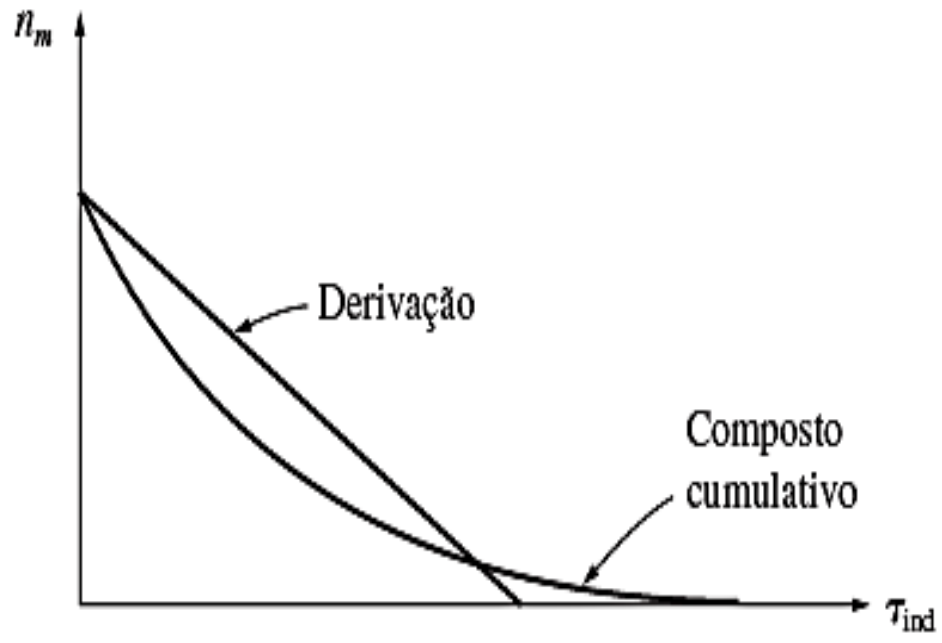

Figura 10 - Curvas téoricas de saída dos motores composto cumulativo e shunt Fonte: Chapman (2013).

\subsubsection{Validação do Motor Composto Diferencial}

O resultado da simulação do motor $\mathrm{CC}$ composto diferencial em derivação longa está ilustrado na Figura 13. Percebe-se que, a vazio, a máquina opera na velocidade nominal de $1800 \mathrm{rpm}$. Esta máquina caracteriza-se por possuir uma interação entre os campos onde as forças magnetomotrizes shunt e série subtraem-se entre si. Assim, com o aumento da carga, a corrente de armadura aumenta e o fluxo do motor diminui. Esta diminuição provoca o aumento da velocidade, no entanto, a elevação da velocidade faz com que a corrente drenada de carga aumente ainda mais, o que amplifica $I_{A}$, diminui o fluxo e, consequentemente, colabora mais uma vez com o aumento da velocidade. O resultado deste processo na rotina computacional desenvolvida é ilustrado na Figura 11. Com o aumento da carga, percebe-se uma elevação cada vez mais brusca na velocidade. 
CASTRO, A. C. S. et al. Ferramenta computacional didática para simulação de máquinas elétricas de corrente contínua de conexão composta com derivação longa

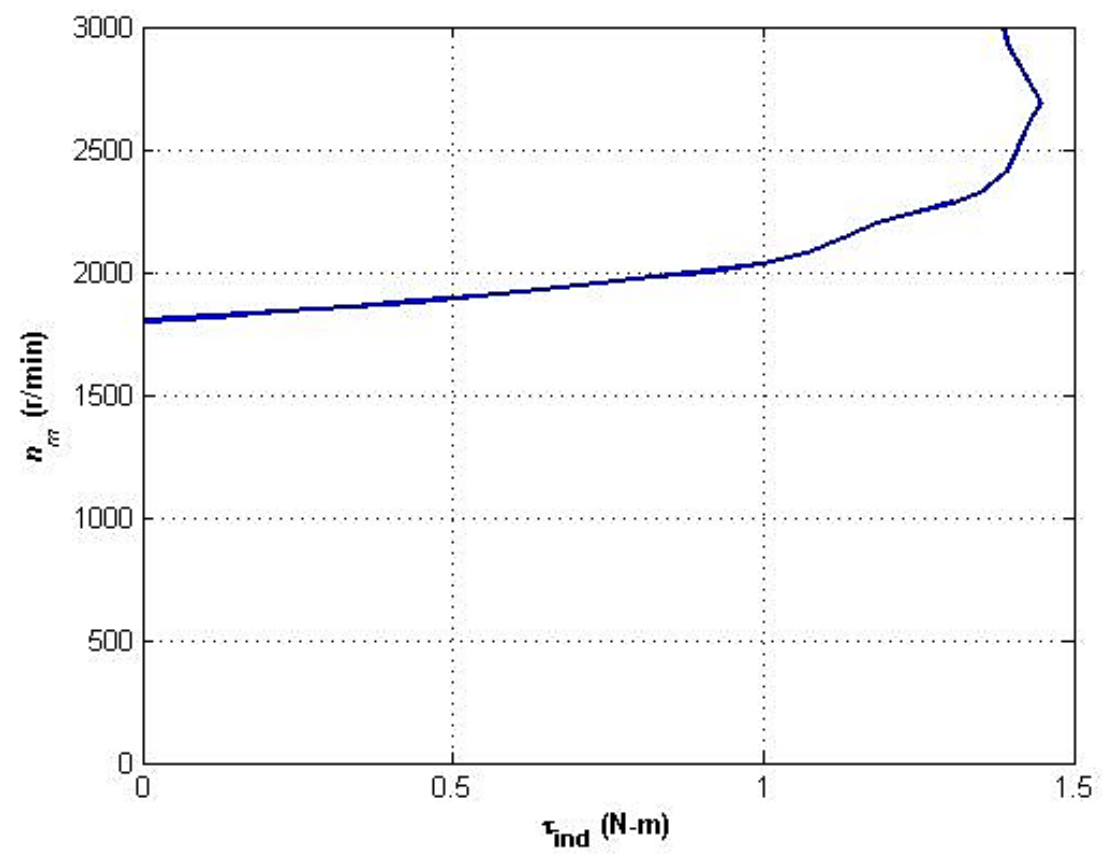

Figura 11 - Simulação da curva de saída do motor composto diferencial Fonte: Autores (2018).

A Figura 12 indica a curva característica de saída teórica para um motor. Percebe-se a semelhança entre esta curva e aquela apresentada na figura anterior. Algumas passagens da curva mostrada na Figura 11 não se mostram tão suaves quanto a da Figura 12, porém, no geral, os comportamentos mostram-se similares. O procedimento de cálculo interno da rotina computacional, através dos dados vetoriais na análise gráfica, faz com que ocorra este tipo de comportamento da curva final.

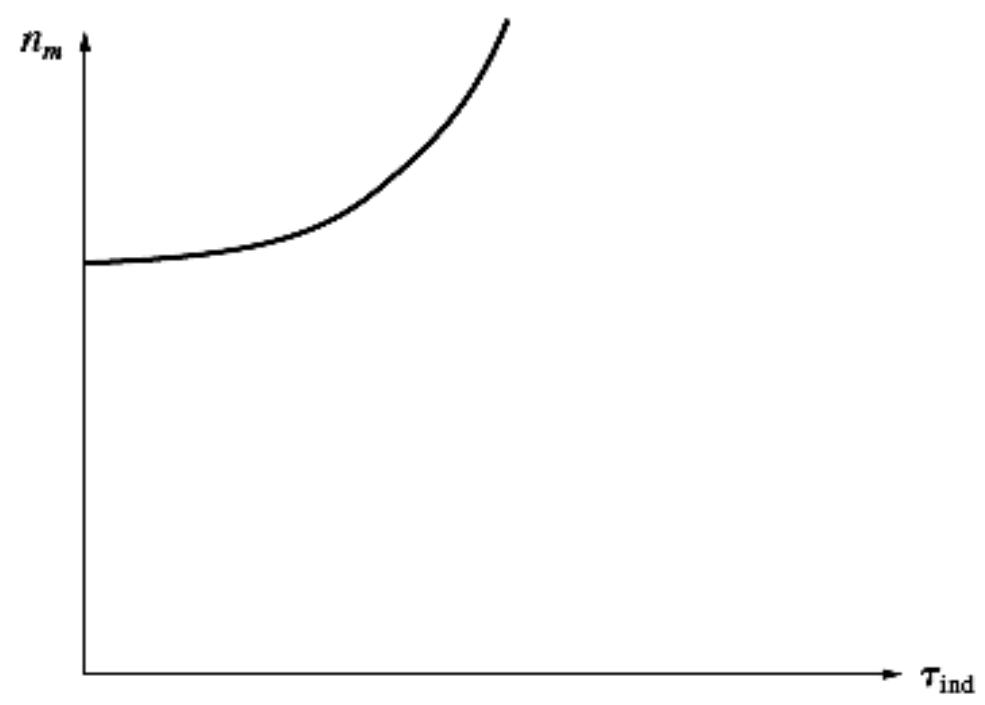

Figura 12 - Curva de saída teórica do motor composto diferencial Fonte: Chapman (2013).

ForSci.: r. cient. IFMG, Formiga, v. 7, n. 1, e00584, jan./jun. 2019. 
CASTRO, A. C. S. et al. Ferramenta computacional didática para simulação de máquinas elétricas de corrente contínua de conexão composta com derivação longa

\subsubsection{Validação do Gerador Composto Cumulativo}

Na Figura 15, consta a simulação das curvas de saída de três geradores compostos cumulativos com shunt longo, cujo número de espiras por polo no enrolamento é de 75, 100 e 150, da curva inferior para a curva superior, respectivamente.

No gerador que possui menos espiras em série, a queda resistiva prevalece e a tensão tende a cair, como verificado na Figura 13, mas não tão acentuadamente quanto um gerador shunt. No gerador que possui valor mediano de espiras, a tensão terminal inicialmente se eleva, mas em seguida decai. Na curva central, representada por pontilhados com asteriscos, da Figura 13, este comportamento pode ser visualizado de forma aproximada. Por fim, no gerador com maior número de espiras em série, a princípio a tensão terminal aumenta com a elevação da carga, e este aumento prevalece por uma faixa maior que o gerador anterior. Assim, verifica-se que, a vazio, as curvas para ambos os geradores se iniciam no valor de tensão nominal da máquina $(220 \mathrm{~V})$, porém, o aumento do número de espiras série faz com que a curva apresente maiores valores de tensão ao longo da variação de carga.

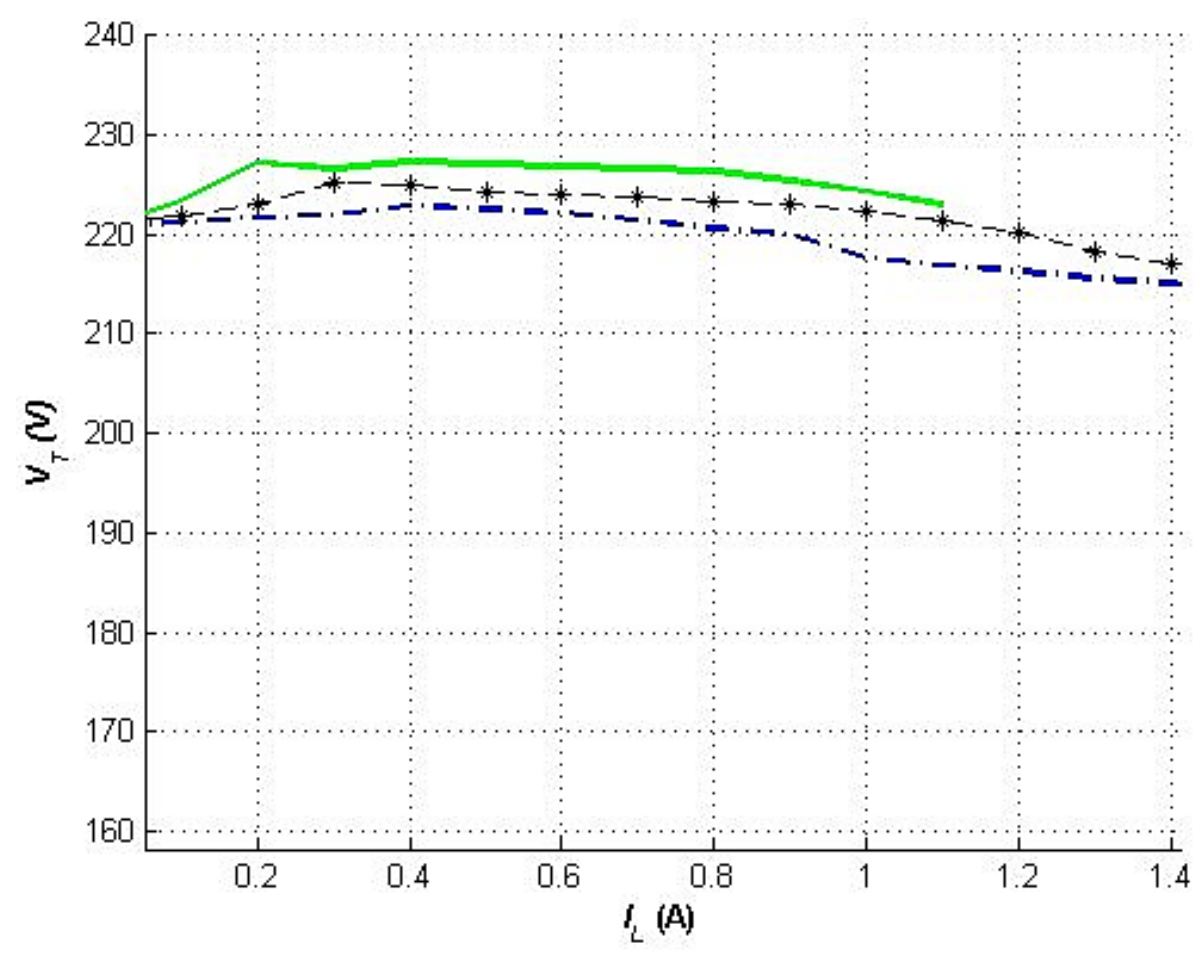

Figura 13 - Simulação das curvas de saída do gerador composto cumulativo com 75 (em azul pontilhada), 100 (em preto com asteriscos) e 150 (em verde contínua) espiras série Fonte: Autores (2018). 
CASTRO, A. C. S. et al. Ferramenta computacional didática para simulação de máquinas elétricas de corrente contínua de conexão composta com derivação longa

A Figura 14 apresenta a característica geral de saída dos geradores hipocomposto, normal e hipercomposto, nos quais a diferença básica consta no número de espiras série, que aumenta do primeiro para o último, respectivamente. Quando a Figura 14 é comparada à Figura 13, percebe-se a semelhança entre ambas, contatando-se que quanto menor o número de espiras série, mais decrescente a curva se torna, o que verifica a validade dos resultados obtidos computacionalmente.

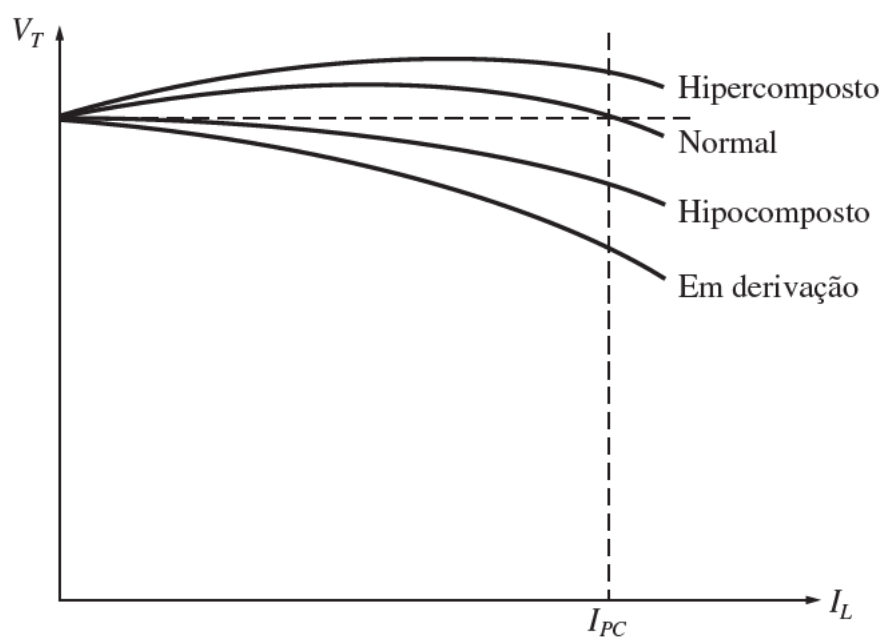

Figura 14 - Curvas teóricas de saída dos geradores composto cumulativos Fonte: Chapmam (2013).

\subsubsection{Validação do Gerador Composto Diferencial}

A Figura 15 indica a simulação do gerador composto diferencial submetida aos dados nominais da máquina (em vermelho) e a curva do gerador shunt para efeito de comparação. No gerador composto diferencial, com o aumento da carga, a tensão tende a cair devido à queda resistiva e também pela diminuição da força magnetomotriz (a força magnetomotriz do campo série aumenta e esta é subtraída da força magnetomotriz do campo shunt). A partir deste efeito de superposição, a tensão da máquina tende a cair drasticamente. A curva em vermelho mostrada na Figura 15 ilustra esta queda. Percebe-se o quão acentuado é o declínio da curva do gerador diferencial, quando comparada a do gerador shunt, e também pode-se observar que ambas partem da mesma tensão a vazio (220 V). 
CASTRO, A. C. S. et al. Ferramenta computacional didática para simulação de máquinas elétricas de corrente contínua de conexão composta com derivação longa

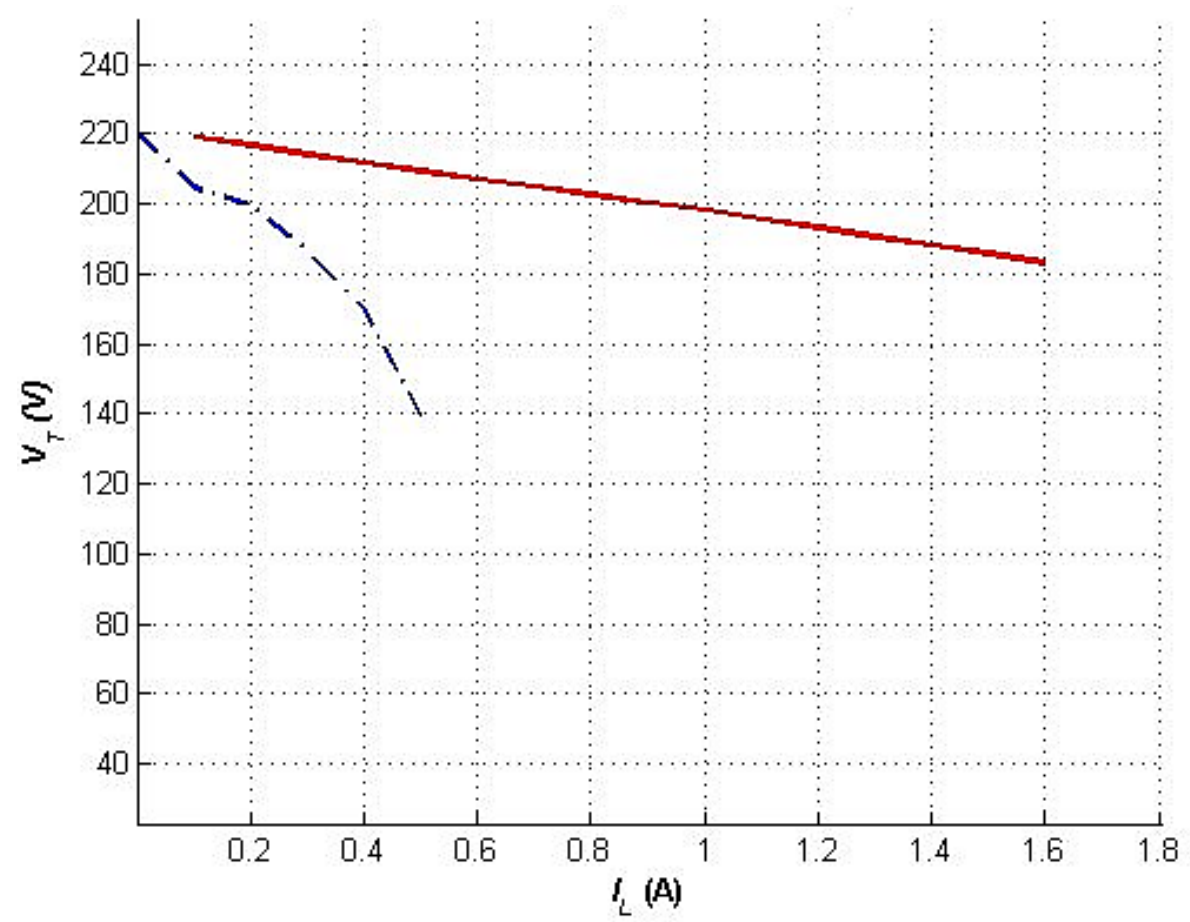

Figura 15 - Simulação das curvas de saída dos geradores composto diferencial (em vermelho contínuo) e shunt (em azul pontilhado)

Fonte: Autores (2018).

A Figura 16 mostra a característica de saída do gerador composto diferencial, em relação à saída do gerador, dada pela referência teórica. É possível constatar o mesmo comportamento apresentado pelas curvas obtidas na simulação.

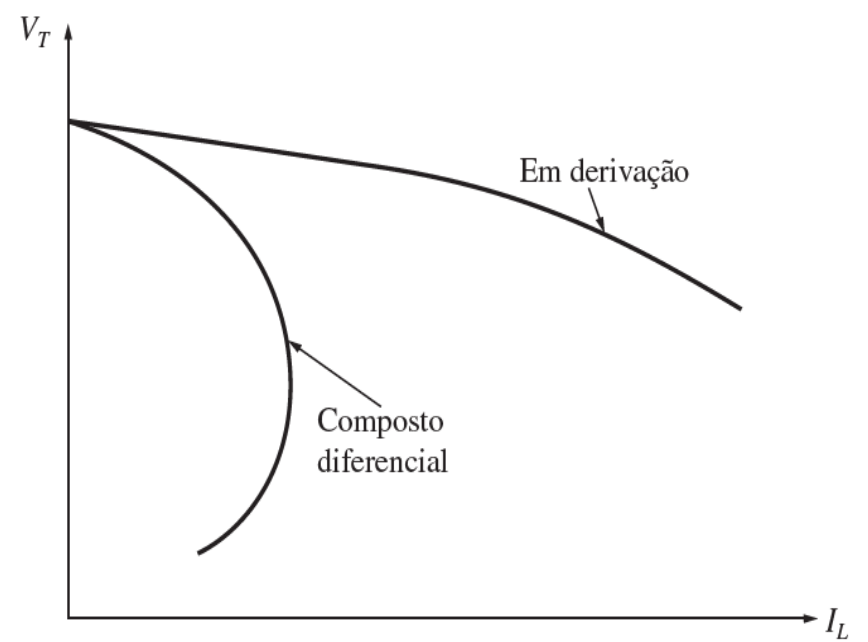

Figura 16 - Curva de saída teórica do gerador composto diferencial Fonte: Chapman (2013). 
CASTRO, A. C. S. et al. Ferramenta computacional didática para simulação de máquinas elétricas de corrente contínua de conexão composta com derivação longa

\title{
3 CONCLUSÃO
}

A ferramenta computacional desenvolvida tem por base equações que modelam o comportamento das máquinas de corrente contínua (geradores e motores) em regime permanente. O objetivo é produzir curvas de interesse prático ao estudo de tais dispositivos. Os resultados de simulações computacionais, gerados com a utilização da ferramenta computacional desenvolvida, mostram uma alta concordância com as curvas obtidas nas principais referências bibliográficas utilizadas no estudo das máquinas elétricas de corrente contínua.

Nos geradores compostos cumulativos, é possível evidenciar a partir da simulação o efeito da variação do número de espiras série, que pode torná-lo hipocomposto, normal ou hipercomposto, o que influencia diretamente na sua regulação de tensão. Para o gerador diferencial, é demostrada a péssima característica de queda de tensão que esta máquina possui (KOSOW, 2000), conforme a possível comparação do comportamento de um gerador shunt nas mesmas condições.

Em relação aos motores, a simulação computacional permitiu a avaliação do desempenho dos motores para condições de carga impossíveis de se reproduzir nas aulas práticas de máquinas elétricas, do Laboratório do IFMG - Campus Formiga. Analisando-se os resultados, observa-se que a relação entre o torque e a velocidade no motor composto cumulativo é inversamente proporcional, ou seja, os maiores valores de torque são obtidos para velocidades menores. Já o motor composto diferencial é caracterizado pela instabilidade durante o aumento de carga, pois a velocidade tende a disparar bruscamente. Este motor apresenta apenas interesse didático, uma vez que não possui aplicação prática. Ademais, os autores pretendem modelar as outras configurações de máquinas de corrente contínua existentes e criar uma interface didática para auxiliar nas aulas de máquinas elétricas em universidades e cursos técnicos.

\section{DIDACTIC COMPUTATIONAL TOOL FOR SIMULATION OF CONTINUOUS CURRENT ELECTRIC MACHINES COMPOSED IN LONG DERIVATION}

\begin{abstract}
The electrical machines are present in almost all the activities that involving the handling of loads in industrial, commercial and residential environments. Thus, the study of the operation of these devices is fundamental for several undergraduate courses, such as the electrical engineering course and the control and automation course, for example. In this context, the
\end{abstract}


CASTRO, A. C. S. et al. Ferramenta computacional didática para simulação de máquinas elétricas de corrente contínua de conexão composta com derivação longa

association between theoretical and practical knowledge assists in the study of students and in the way teachers teach. However, it is often not possible to perform all theoretical situations in laboratories. In these cases, the use of computational simulation appears as an efficient tool, able to present the fundamental curves for the study of the machines in a didactic way and for all the existing load conditions. In this way, this article presents results of computational simulation of electric machines of direct current (motors and generators), realized in the tool $M A T L A B \circledR$, in the compound configuration in long derivation. The model developed is based on the permanent regime equations of such equipment and the objective is to obtain practical curves, easily found in the main literature of the field. The computational tool developed is validated by means of comparison with curves of the literature presenting satisfactory agreement.

Keywords: Didactic tool. Electric current machines. MATLAB®.

\section{REFERÊNCIAS}

ALVARADO, M. S. Modelo matemático de un motor de corriente contínua separadamente excitado: control de velocidad por corriente de armadura. Latin American Journal of Physics Education, Vol. 6, No. 1, March 2012.

BELHOT, R. V.; FIGUEIREDO, R. S.; MALAVÉ, C. O. O uso da simulação no ensino de engenharia. Anais do Congresso Brasileiro de Educação em Engenharia. Porto Alegre, RS. 2001.

CHAPMAN, S. J. Fundamentos de máquinas elétricas. 5. ed. Porto Alegre: Bookman, 2013.

CRESPO, D. L.; GUINGLA, D. A. Modelamiento y simulación de un motor/generador eléctrico de corriente continua controlado por campo/armadura y con carga. Espol, p. 15. 2010.

DEL TORO, V. Fundamentos de máquinas elétricas. 2. ed. São Paulo: LTC, 1999.

FARIAS, A. T. Modelo dinâmico de motores de indução como instrumento para o ensino de maquinas elétricas. 2017. 76f. Dissertação (Mestrado Profissional em Automação e Controle de Processos) - Programa de Pós-Graduação em Automação e Controle de Processos, Instituto Federal de Educação, Ciência e Tecnologia de São Paulo, São Paulo. 2017.

FITZGERALD, A. E.; KINGSLEY JR., C.; UMANS, S. D. Máquinas elétricas: com introdução à eletrônica de potência. 7. ed. Porto Alegre: Bookman. 2014.

HAMBLEY, A. R. Electrical engineering, principles and applications. New Jersey: Pearson, 2014. 
CASTRO, A. C. S. et al. Ferramenta computacional didática para simulação de máquinas elétricas de corrente contínua de conexão composta com derivação longa

KHALIL, F. R. O uso da tecnologia de simulação na prática docente do ensino superior. XVI Encontro Nacional de Didática e Práticas de Ensino, UNICAMP. Campinas - SP. 2012.

KOSOW, I. L. Máquinas elétricas e transformadores. Rio de Janeiro: Globo, 2000.

LODER, L. L.; BENDER. F. A. O uso de programas de simulação em cursos de engenharia: possibilidades e necessidades. Anais XXXV Congresso Brasileiro de Educação em Engenharia. Curitiba, PR: Associação Brasileira de Educação de Engenharia, 2007.

MARCHETTO FILHO, G.; PINHEIRO, D. D.; CARATI, E. G. Ambiente de Simulação de Acionamento de Motores de Indução com Controle Vetorial. Anais XLIV Congresso Brasileiro de Educação em Engenharia. UFRN: Associação Brasileira de Educação de Engenharia, 2016.

MELO, B. M. S. Modelagem e Simulação de uma Máquina Elétrica de Corrente Contínua levando-se em Consideração os Efeitos de Reação de Armadura. 2006.

Dissertação de Mestrado- Programa de Pós-Graduação em Engenharia de Telecomunicação), Universidade de São Paulo. São Paulo, SP. 2006.

PEREIRA, L. S.; MONTENEGRO, L. C.; AMORA, M. A. B.; MACHADO, I. R. Melhorias Pedagógicas nas Disciplinas de Máquinas Elétricas, Conversão Eletromecânica de Energia e Máquinas Síncronas. Anais XLIV Congresso Brasileiro de Educação em Engenharia. UFRN: Associação Brasileira de Educação de Engenharia, 2016.

YILDIZ, A. B. Electrical equivalent circuit based modeling and analysis of direct current motors. International Journal of Electrical Power \& Energy Systems, vol. 43, issue 1, December 2012, pages 1043-1047. DOI: https://doi.org/10.1016/j.ijepes.2012.06.063

\section{DADOS DOS AUTORES:}

\section{Ana Carolina Silva Castro}

E-mail: anacarolinasilvacastro@yahoo.com.br

Currículo Lattes: http://lattes.cnpq.br/9475741339163808Graduação em Engenharia Elétrica pelo Instituto Federal de Educação, Ciência e Tecnologia de Mina Gerais (IFMG), Campus Formiga. Trabalha atualmente no Grupo Gomide com o cargo de engenharia eletricista, sendo responsável técnica de execução de obras. Também é pesquisadora do Grupo de Pesquisa CNPq GSE (Grupo de Soluções em Engenharia).

\section{Mariana Guimarães dos Santos}

E-mail: mariana.santos@ifmg.edu.br

Currículo Lattes: http://lattes.cnpq.br/4661598896242893

Mestrado em Engenharia Elétrica pelo Programa de Pós Graduação em Elétrica - Associação ampla entre Universidade Federal de São João Del Rei (UFSJ) e Centro Federal de Educação 
CASTRO, A. C. S. et al. Ferramenta computacional didática para simulação de máquinas elétricas de corrente contínua de conexão composta com derivação longa

Tecnológica (CEFET-MG) e graduação em Engenharia Elétrica pela UFSJ. Atualmente é professora do curso de Engenharia Elétrica do IFMG - Campus Formiga e suas áreas de interesse são Circuitos Elétricos e Magnéticos e Máquinas Elétricas e Acionamentos, assim como Ensino de Engenharia e suas Tecnologias. Também é pesquisadora do Grupo de Pesquisa CNPq GSE (Grupo de Soluções em Engenharia).

\section{Patrick Santos de Oliveira}

E-mail: patrick.oliveira@ifmg.edu.br

Currículo Lattes: http://lattes.cnpq.br/2186926510200941

Doutorando em Engenharia Elétrica pela Universidade Federal de Juiz de Fora (UFJF), mestrado em Engenharia Elétrica pela Universidade de São Paulo (USP) - Escola de Engenharia de São Carlos e graduação em Engenharia Elétrica pela Fundação Educacional de Barretos. Atualmente é professor do curso de Engenharia Elétrica IFMG - Campus Formiga e suas áreas de interesse são Qualidade da Energia Elétrica, eficiência de energia e proteção de sistemas elétricos de potência.

\section{Ana Paula Lima dos Santos}

E-mail: anapaula.lima@ifmg.edu.br

Currículo Lattes: http://lattes.cnpq.br/3649720412754134

Mestrado em Engenharia Elétrica pelo Programa de Pós Graduação em Elétrica - Associação ampla entre UFSJ e CEFET-MG e graduação em Engenharia Elétrica pela UFSJ. Atualmente é professora do curso de Engenharia Elétrica do IFMG - Campus Formiga e suas áreas de interesse são Máquinas Elétricas, Sistemas Embarcados, Modelagem de Sistemas, assim como Ensino de Engenharia e suas Tecnologias. Também é pesquisadora do Grupo de Pesquisa CNPq GSE (Grupo de Soluções em Engenharia).

\section{Gustavo Lobato Campos}

E-mail: gustavo.lobato@ifmg.edu.br

Currículo Lattes: http://lattes.cnpq.br/9613750934178733

Doutorado em Engenharia Nuclear pela Universidade Federal de Minas Gerais (UFMG), Mestrado em Engenharia Elétrica pela PUC Minas e graduação em Engenharia Eletrônica e de Telecomunicações pela PUC Minas. Atualmente é professor do curso de Engenharia Elétrica e Técnico em Eletrotécnica do IFMG - Campus Formiga e suas áreas de interesse são Sistemas Embarcados, Eletromagnetismo e Compatibilidade Eletromagnética, assim como Ensino de Engenharia e suas Tecnologias. Também é coordenador do Grupo de Pesquisa CNPq GSE (Grupo de Soluções em Engenharia). Possui também experiência no setor privado em empresas do setor automotivo como Siemens VDO e Continental AG. 